Vietnam Journal of Mechanics, VAST, Vol.40, No. 1 (2018), pp. 89-103

DOI:10.15625/0866-7136/10579

\title{
STATIC AND FREE VIBRATION ANALYSES OF LAMINATED COMPOSITE SHELLS BY CELL-BASED SMOOTHED DISCRETE SHEAR GAP METHOD (CS-DSG3) USING THREE-NODE TRIANGULAR ELEMENTS
}

\author{
Pham Quoc Hoa ${ }^{1,2}$, Tran The Van ${ }^{2}$, Pham Tien Dat ${ }^{1}$, Dang Trung Hau ${ }^{3}$, \\ Nguyen Viet $\mathrm{Ha}^{1}$, Nguyen Manh Hung ${ }^{2}$, Nguyen Thoi Trung, \\ ${ }^{1}$ Le Quy Don University, Hanoi, Vietnam \\ ${ }^{2}$ Tran Dai Nghia University, Ho Chi Minh City, Vietnam \\ ${ }^{3}$ Ton Duc Thang University, Ho Chi Minh City, Vietnam \\ *E-mail: nguyenthoitrung@tdt.edu.vn \\ Received July 30, 2017
}

\begin{abstract}
A cell-based smoothed discrete shear gap method (CS-DSG3) using three-node triangular elements was recently proposed to improve the performance of the discrete shear gap method (DSG3) for static and free vibration analyses of isotropic ReissnerMindlin plates and shells. In this paper, the CS-DSG3 is further extended for static and free vibration analyses of laminated composite shells. In the present method, the firstorder shear deformation theory (FSDT) is used in the formulation due to the simplicity and computational efficiency. The accuracy and reliability of the proposed method are verified by comparing its numerical solutions with those of others available numerical results.

Keywords: Smoothed finite element methods (S-FEM), cell-based smoothed discrete shear gap method (CS-DSG3), laminated composite shell, first-order shear deformation theory (FSDT).
\end{abstract}

\section{INTRODUCTION}

Owning many superior properties as high strength-to-weight and high stiffnessto-weight ratios, excellent fatigue strength, etc., composite materials have been widely used in plate and shell structures in many engineering fields such as naval, automotive, aerospace, defense industries and many other areas. Many methods for analysis of the laminated composite plate and shell have been developed recently. For example, A. Bhimaraddi has proposed a three-dimensional (3D) elasticity solution for static and vibration of double curved shallow shell made of composite material [1,2]. In this study, the shell thickness is divided into layers of smaller thickness, which can help increase the accuracy in analysis of thickness shell. However, the computational cost for 3D analysis

(C) 2018 Vietnam Academy of Science and Technology 
is still much higher than that for two-dimensional (2D) analysis. Therefore, a 2D model was preferred for analysis of the laminated composite shell and attracted the concern of many researchers. For example, K. P. Rao [3] developed a rectangular laminated shell element. In these papers, the authors only used the classical laminated theory (CLT) which completed neglect the shear deformation effect, and hence had a negative influence to the accuracy of analysis results of thickness shell. To overcome the drawbacks, the first order shear deformation theory (FSDT) was used to analyze for the laminated shell. J. N. Reddy [4] presented a development of exact solutions based on the Sander shell theory for the double curved shell. S. J. Hossain et al. [5] developed a four node quadrilateral isoparametric element using mixed interpolation of tensorial components (MITC) approach. D. Chakravorty et al. [6] proposed an eight node curved quadrilateral isoparametric element for the vibration analysis of double curved laminated composite shells. The FSDT, however, the accuracy of solutions strongly depends on shear correction factors to ensure the stability of the solution [7]. Hence, the higher order shear deformation (HSDT), layerwise (LWT) or zigzag (ZIGT) theories have been proposed to analyze the laminated composite shell. For instance, L. Librescu and A. A. Khdeir [8,9] used the state space concept conjunction of the Lévy method for static and free vibration analyses of the laminated composite shell. J. N. Reddy and C. F. Liu [10] developed a HSDT for the laminated composite shell. In this study, the Navier-type exact solutions for static and free vibration analyses were presented for spherical and cylindrical shells. R. K. Khare et al. [11] presented a 2D HSDT for analysis of laminated composite and sandwich shallow shells subjected to thermal and mechanical loads. To achieve more accurate results for laminated shell, M. Y. Yasin [12] proposed a four-node quadrilateral element for static and free vibration analyses of laminated shallow shells based on the ZIGT. G. Giunta et al. [13] mixed the HSDT with LWT and ZIGT for analysis of the laminated double curved shell. A. J. M. Ferreira et al. [14] studied the radial basis functions (RBFs) collocation based on a LWT for the static and free vibration analyses of the laminated shells. From above literature review, it is obvious that, HSDT, LWT and ZIGT have been achieved the great interest from researchers. However, they have a limitation in computational cost which causes the limit of their practical applications. In addition, in recent years, many promising computational approaches have also been proposed for analyzing plate/shell problems. For example, Chien H. Thai et al. [15-17] developed isogeometric analysis for static, free vibration, and buckling analysis of laminated composite plates. T. Rabczuk et al. $[18,19]$ used meshfree method based on the Kirchhoff-Love (KL) theory to investigate for crack and fluid-structure interaction of thin shells. N. Nguyen-Thanh et al. [20,21] presented isogeometric analysis to study for thin shell structures.

On the other hand, in front of the development of numerical methods, Liu and Nguyen-Thoi [22] integrated the strain smoothing technique into the finite element method to create a series of smoothed finite element methods (S-FEM) including the cellbased smoothed finite element (CS-FEM) [23-28], the node-based smoothed finite element (NS-FEM) [29-31], the edge-based smooth finite element method (ES-FEM) [32,33], and the face-based smoothed finite element (FS-FEM) [34]. Each of these smoothed finite element methods has different properties and has been used to produce desired solutions 
for a wide class of benchmark and practical mechanics problems. The smoothed finite element methods have also been further investigated and applied to various problems as plates and shells [35-43], and some other applications.

Among the S-FEM models, the CS-FEM has shown some interesting properties in the solid mechanics problems. Extending the idea of the CS-FEM to plate structures, Nguyen-Thoi et al. [43] have recently formulated a cell-based smoothed stabilized discrete shear gap element (CS-DSG3) for static and free vibration analyses of isotropic shell structures by combining the CS-FEM with the original DSG3 [44]. In the CS-DSG3, each three-node triangular element will be divided into three sub-triangles, and in each subtriangle, the stabilized DSG3 is used to compute the strains. Following that the strain smoothing technique on whole triangular element is used to smooth the strains on three sub-triangles. The numerical results have shown that the CS-DSG3 is free of shear locking and achieves a high accuracy compared with the exact solutions and other existing elements in the literature.

This paper aims to extend further the CS-DSG3 to static and free vibration analyses of the laminate composite shell. The FSDT and flat shell theory are used in the formulation due to the simplicity and computational efficiency. The accuracy and reliability of the proposed method are verified by comparing its numerical solutions with those of others available numerical results.

\section{WEAK FORM OF LAMINATED COMPOSITE SHELL}

A shell is a 3D structure and it is often convenient to define the geometry of shell structures in the global coordinate system. Based on the theories of formulation [45], shell elements can be classified into three main groups: (1) degenerated shell elements derived from the 3D solid theory; (2) curved shell elements based on general shell theory; and (3) flat shell elements formulated by combining a plane elastic membrane elements (plane stress elements) and a plate bending elements. Among these three groups, the flat shell elements are more popular due to simple formulation and low computational cost, and hence the theory of flat shell elements will be chosen to consider in this study.

To generate the element stiffness matrix for the membrane and plate bending elements, the elements must be defined in a local plane. Thus it is necessary to use local coordinates for computing the element mass, stiffness matrices and load vectors of the flat shell elements. In this case, a transformation between global and local coordinates is required and can be defined by using direction cosines. Based on the FSDT and flat shell theory, the standard weak-form Galerkin of shell problem is defined by

$$
\int_{\Omega} \delta \mathbf{u}^{T} \mathbf{m u ̈} \mathrm{d} \Omega+\int_{\Omega} \delta\left[\begin{array}{c}
\boldsymbol{\varepsilon}_{m}^{T} \\
\boldsymbol{\kappa}^{T} \\
\boldsymbol{\gamma}^{T}
\end{array}\right]^{T}\left[\begin{array}{ccc}
\mathbf{D}_{m} & \mathbf{D}_{m b} & 0 \\
\mathbf{D}_{m b} & \mathbf{D}_{b} & 0 \\
0 & 0 & \mathbf{D}_{s}
\end{array}\right]\left[\begin{array}{c}
\boldsymbol{\varepsilon}_{m} \\
\boldsymbol{\kappa} \\
\boldsymbol{\gamma}
\end{array}\right] \mathrm{d} \Omega=\int_{\Omega} \delta \mathbf{u}^{T} \mathbf{b} \mathrm{d} \Omega,
$$

where $\mathbf{u}=\left\{u_{0}, v_{0}, w_{0}, \beta_{x}, \beta_{y}, \beta_{z}\right\}^{T}$ is the displacement field at any point on the middle plane of shell with $u_{0}, v_{0}, w_{0}$ and $\beta_{x}, \beta_{y}, \beta_{z}$ denote the displacement components in the 
$x, y, z$ directions, respectively; $\mathbf{b}$ is an applied load vector; $\boldsymbol{\varepsilon}_{m}, \boldsymbol{\kappa}$ and $\boldsymbol{\gamma}$ are defined by

$\boldsymbol{\varepsilon}_{m}=\left\{\begin{array}{lll}u_{0, x} & v_{0, y} & u_{0, y}+v_{0, x}\end{array}\right\}^{T}, \boldsymbol{\kappa}=\left\{\begin{array}{lll}\beta_{x, x} & \beta_{y, y} & \beta_{x, y}+\beta_{y, x}\end{array}\right\}^{T}, \boldsymbol{\gamma}=\left\{\begin{array}{ll}w_{0, x}+\beta_{x} & w_{0, y}+\beta_{y}\end{array}\right\}^{T}$.

In Eq. (1), $\mathbf{D}_{m}, \mathbf{D}_{b}, \mathbf{D}_{m b}$ and $\mathbf{D}_{s}$ are the extensional, bending, bending-extension coupling stiffness, respectively, which are given by

$$
\begin{aligned}
& \left(\mathbf{D}_{m}, \mathbf{D}_{m b}, \mathbf{D}_{b}\right)=\int_{-h / 2}^{h / 2}\left(1, z, z^{2}\right) \bar{Q}_{i j} \mathrm{~d} z, \quad(i, j=1,2,6) \\
& \mathbf{D}_{s}=\int_{-h / 2}^{h / 2} \kappa \bar{Q}_{i j} \mathrm{~d} z, \quad(i, j=4,5)
\end{aligned}
$$

where $h$ is the thickness of the shell; $\kappa=5 / 6$ is shear coefficient; $\bar{Q}_{i j}$ are the transformed material constants of the $k^{\text {th }}$ lamina [7]; $\mathbf{m}$ is the mass matrix containing the mass density of the material $\rho$, expressed by

$$
\mathbf{m}=\sum_{k=1}^{N} \rho^{k} \int_{z_{k}}^{z_{k+1}}\left[\begin{array}{cccccc}
1 & 0 & 0 & z & 0 & 0 \\
0 & 1 & 0 & 0 & z & 0 \\
0 & 0 & 1 & 0 & 0 & 0 \\
z & 0 & 0 & z^{2} & 0 & 0 \\
0 & z & 0 & 0 & z^{2} & 0 \\
0 & 0 & 0 & 0 & 0 & 0
\end{array}\right] \mathrm{d} z
$$

\section{CS-DSG3 FORMULATION FOR LAMINATED COMPOSITE SHELL}

In the DSG3 [44], the shear strain is linear interpolated based on the concept "shear gap" of displacement along the sides of the elements by using the standard element shape functions. Accordingly, the approximation $\mathbf{u}_{e}$ of a 3-node triangular shell element $\Omega_{e}$ can be written as

$$
\mathbf{u}_{e}=\sum_{I=1}^{3} N_{I}(\mathbf{x}) \mathbf{I}_{6} \mathbf{d}_{e I}=\sum_{I=1}^{3} \mathbf{N}_{I} \mathbf{d}_{e I},
$$

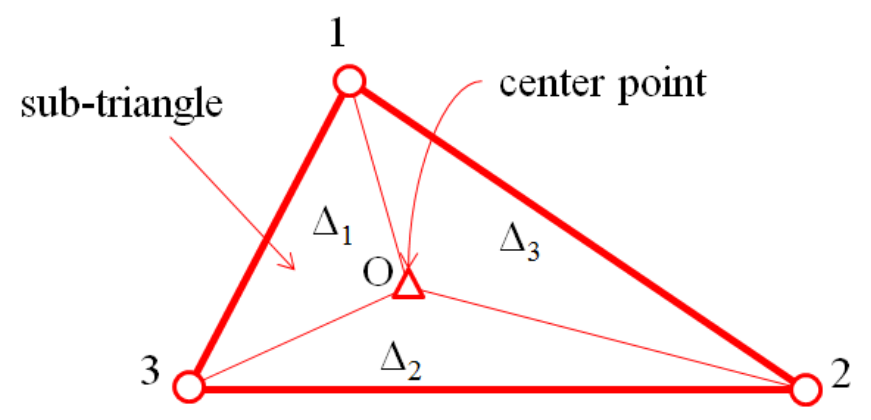

Fig. 1. Three sub-triangles created from the triangle 1-2-3 in CS-DSG3 by connecting the central point $\mathrm{O}$ with three field nodes 1,2 and 3 
where $\mathbf{d}_{e I}=\left\{u_{I}, v_{I}, w_{I}, \beta_{x I}, \beta_{y I}, \beta_{z I}\right\}^{T}$ is the nodal degrees of freedom associated with the Ith node and $N_{I}(\mathbf{x})$ is linear shape functions in a natural coordinate defined by

$$
N_{1}=1-\xi-\eta ; \quad N_{2}=\xi ; \quad N_{3}=\eta .
$$

Then, the membrane, bending and shear strains in the element are then obtained by

$$
\begin{aligned}
& \boldsymbol{\varepsilon}_{m}=\left[\mathbf{B}_{m 1}, \mathbf{B}_{m 2}, \mathbf{B}_{m 3}\right] \mathbf{d}_{e}=\mathbf{B}_{m} \mathbf{d}_{e}, \\
& \boldsymbol{\kappa}=\left[\mathbf{B}_{b 1}, \mathbf{B}_{b 2}, \mathbf{B}_{b 3}\right] \mathbf{d}_{e}=\mathbf{B}_{b} \mathbf{d}_{e}, \\
& \boldsymbol{\gamma}=\left[\mathbf{B}_{s 1}, \mathbf{B}_{s 2}, \mathbf{B}_{s 3}\right] \mathbf{d}_{e}=\mathbf{B}_{s} \mathbf{d}_{e},
\end{aligned}
$$

where $\mathbf{B}_{m i}, \mathbf{B}_{b i}$ and $\mathbf{B}_{s i}$ are determined as $\mathbf{R}_{i}, \mathbf{B}_{i}$ and $\mathbf{S}_{i}$ in [43].

The global stiffness matrix now can be written by

$$
\mathbf{K}^{D S G 3}=\sum_{e=1}^{N_{n}} \mathbf{K}_{e}^{D S G 3},
$$

where $\mathbf{K}_{e}^{D S G 3}$ is the element stiffness matrix of the DSG3 element and is given by

$$
\mathbf{K}_{e}^{D S G 3}=\mathbf{T}^{T}\left(\int_{\Omega_{e}}\left\{\begin{array}{c}
\mathbf{B}_{m} \\
\mathbf{B}_{b} \\
\mathbf{B}_{s}
\end{array}\right\}^{T}\left[\begin{array}{ccc}
\mathbf{D}_{m} & \mathbf{D}_{m b} & \mathbf{0} \\
\mathbf{D}_{m b} & \mathbf{D}_{b} & \mathbf{0} \\
\mathbf{0} & \mathbf{0} & \mathbf{D}_{s}
\end{array}\right]\left\{\begin{array}{c}
\mathbf{B}_{m} \\
\mathbf{B}_{b} \\
\mathbf{B}_{s}
\end{array}\right\} \mathrm{d} \Omega\right) \mathbf{T},
$$

in which $\mathbf{T}$ is the transformation matrix for whole element that is defined by

$$
\mathbf{T}=\operatorname{diag}\left(\mathbf{T}_{0}, \mathbf{T}_{0}, \mathbf{T}_{0}\right),
$$

and $\mathbf{T}$ is a transformation matrix at each point [45].

In the CS-DSG3, each triangular element was divided into three sub-triangles, $\Delta_{j}$, by connecting the central point of the element to three field nodes as shown in Fig. 1. Then the displacement vector at central point was assumed to be the simple average of three displacement vectors of three field nodes. To avoid the shear locking phenomenon, in each sub-triangles, the stabilized DSG3 was used to compute the strain fields. The detail formulation of CS-DSG3 can be found in references $[43,46]$. by

The smoothed membrane, bending and shear strains in the CS-DSG3 are expressed

$$
\boldsymbol{\varepsilon}_{m}=\widetilde{\mathbf{B}}_{m} \mathbf{T} \mathbf{d}_{e}, \quad \boldsymbol{\kappa}=\widetilde{\mathbf{B}}_{b} \mathbf{T} \mathbf{d}_{e}, \quad \boldsymbol{\gamma}=\widetilde{\mathbf{B}}_{s} \mathbf{T} \mathbf{d}_{e},
$$

where $\widetilde{\mathbf{B}}_{m}, \widetilde{\mathbf{B}}_{b}$ and $\widetilde{\mathbf{B}}_{s}$ are, respectively, the smoothed membrane, bending and shear gradient matrices expressed by

$$
\widetilde{\mathbf{B}}_{k}=\frac{1}{A_{e}} \sum_{i=1}^{3} A_{\Delta_{i}} \mathbf{B}_{k}^{\Delta_{i}}, \quad k=m, b, s
$$

where $A_{e}$ and $A_{\Delta_{i}}$ are the area of element and sub-triangle $\Delta_{i}$, respectively; $\mathbf{B}_{m}^{\Delta_{j}}, \mathbf{B}_{b}^{\Delta_{j}}, \mathbf{B}_{s}^{\Delta_{j}}$ $(j=1,2,3)$ are computed similarly as the matrices $\mathbf{B}_{m}, \mathbf{B}_{b}, \mathbf{B}_{s}$ of the DSG3 in Eqs. (7), but with two following changes: 1$)$ the coordinates of three node $\mathbf{x}_{i}=\left[\begin{array}{ll}x_{i} & y_{i}\end{array}\right]^{T}, i=1,2,3$ are replaced by three nodes of sub-triangle $\Delta_{j}$, respectively; and 2) the area $A_{e}$ is replaced by the area $A_{\Delta_{j}}$ of sub-triangle $\Delta_{j}$. These computational details can be found in [43] By 
substituting Eq. (11) into Eq. (1), the equilibrium equation for the laminated shell is now expressed in the form of

$$
\mathbf{M} \ddot{\mathbf{d}}+\mathbf{K d}=\mathbf{F} \text {, }
$$

in which $\mathbf{M}$ and $\mathbf{K}$ are the global mass and stiffness matrices, $\mathbf{F}$ is the global load vector. They are obtained by assembling from local matrices and expressed as follows

$$
\begin{gathered}
\mathbf{M}=\sum_{e=1}^{N_{e}} \mathbf{M}_{e}=\sum_{e=1}^{N_{e}} \int_{\Omega_{e}} \mathbf{T}^{T} \mathbf{N}^{T} \mathbf{m} \mathbf{N T} \mathrm{d} \Omega, \\
\mathbf{K}=\sum_{e=1}^{N_{e}} \mathbf{K}_{e}^{\mathrm{CS}-\mathrm{DSG} 3}=\sum_{e=1}^{N_{e}} \int_{\Omega_{e}} \mathbf{T}^{T}\left\{\begin{array}{ccc}
\widetilde{\mathbf{B}}_{m}^{T} & \widetilde{\mathbf{B}}_{b}^{T} & \widetilde{\mathbf{B}}_{s}^{T}
\end{array}\right\}\left[\begin{array}{ccc}
\mathbf{D}_{m} & \mathbf{D}_{m b} & 0 \\
\mathbf{D}_{m b} & \mathbf{D}_{b} & 0 \\
0 & 0 & \mathbf{D}_{s}
\end{array}\right]\left\{\begin{array}{l}
\widetilde{\mathbf{B}}_{m}^{T} \\
\widetilde{\mathbf{B}}_{b}^{T} \\
\widetilde{\mathbf{B}}_{s}^{T}
\end{array}\right\} \mathbf{T} \Omega, \\
\mathbf{F}=\sum_{e=1}^{N_{e}} \mathbf{F}_{e}=\sum_{e=1}^{N_{e}} \int_{\Omega_{e}} \mathbf{T}^{T} \mathbf{N}^{T} \mathbf{b} \mathrm{d} \Omega,
\end{gathered}
$$

From Eq. (2), we can see the independence between the strain components and the drilling degree of freedom, $\beta_{z}$. This is the cause of singularity in the global stiffness matrix when all the elements meeting at node are coplanar and there is no coupling between the membrane stiffness and bending stiffness of the element. To deal this problem, the null values of the stiffness matrix corresponding to $\beta_{z}$ are replaced by approximate values. This approximate value is taken to be equal to $10^{-3}$ times the maximum diagonal value in the element stiffness matrix [43].

Note that while the accuracy of the CS-DSG3 [46] and that of the ES-DSG3 [39] are almost the same [46], the CS-DSG3 has lower computational cost. It is because the CSDSG3 only requires the local computation located inside the element which is much more convenient than the ES-DSG3. This advantage of the CS-DSG3 is even further promoted for shell elements

\section{NUMERICAL RESULTS}

In this section, the static and free-vibration analyses of laminated composite spherical and cylindrical shells as shown in Fig. 2 are conducted using the proposed method CS-DSG3. In static analysis, these shells are assumed to be subjected to uniform distributed, sinusoidal and concentrated loads. The effects of the boundary conditions, length to radius ratio and fiber direction on behavior of these shells are considered. The obtained results are compared to the other existing numerical solutions to show the accuracy and stability of the CS-DSG3 in laminated shell analyses. For the convenient comparison, the non-dimensional central deflection and natural frequencies are introduced by

$$
\bar{w}=\frac{1000 w(a / 2, b / 2,0) t^{3} E_{2}}{P a^{4}}, \quad \bar{\omega}=\omega\left(a^{2} / h\right) \sqrt{\rho / E^{2}} .
$$




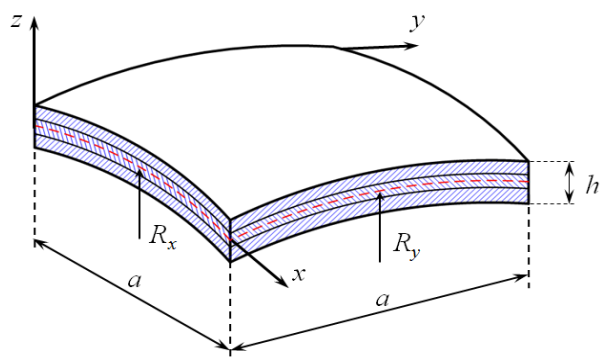

(a) Spherical shell model $\left(R_{x}=R_{y}=R\right)$

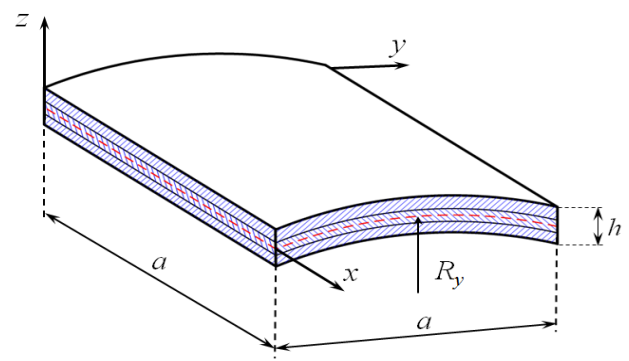

(b) Cylindrical shell model $\left(R_{x}=\infty, R_{y}=R\right)$

Fig. 2. Geometry for laminated composite shells

\subsection{Static analysis}

\subsubsection{Laminated spherical shell}

Firstly, the static analysis of a simply supported laminated spherical shell is studied. The shell composes of several layers such as $\left[0^{\circ} / 90^{\circ}\right],\left[0^{\circ} / 90^{\circ} / 0^{\circ}\right],\left[0^{\circ} / 90^{\circ} / 90^{\circ} / 0^{\circ}\right]$. All the plies have the same thickness and material with mechanical properties given by: $E_{1}=$ $25 E_{2}, G_{12}=0.5 E_{2}, G_{13}=0.5 E_{2}, G_{23}=0.2 E_{2}, v_{12}=0.25$. Tab. 1 presents the nondimensional central deflections of the laminated shell subjected to sinusoidal loading, by the CS-DSG3 in comparison with those by the DSG3 [44] and Reddy and Liu [10] using the FSDT. Further, the non-dimensional central displacements of laminated shell under

Table 1. The non-dimensional center deflections of the laminated spherical shells under sinusoidal load

\begin{tabular}{|c|c|c|c|c|c|c|c|}
\hline \multirow{2}{*}{$R / a$} & \multirow{2}{*}{ Theory } & \multicolumn{2}{|c|}{$0^{\circ} / 90^{\circ}$} & \multicolumn{2}{|c|}{$0^{\circ} / 90^{\circ} / 0^{\circ}$} & \multicolumn{2}{|c|}{$0^{\circ} / 90^{\circ} / 90^{\circ} / 0^{\circ}$} \\
\hline & & \multicolumn{6}{|c|}{$a / h=10 a / h=100 a / h=10 a / h=100 a / h=10 a / h=100$} \\
\hline \multirow{5}{*}{5} & Reddy and Liu FSDT [10] & 11.4290 & 1.1948 & 6.4253 & 1.0337 & 6.3623 & 1.0279 \\
\hline & DSG3 $(24 \times 24)$ & 11.2516 & 1.1630 & 6.3442 & 1.0087 & 6.2653 & 0024 \\
\hline & CS-DSG3 $(8 \times 8)$ & 10.7714 & 1.1287 & 6.1245 & 0.9772 & 6.0546 & 0.9713 \\
\hline & CS-DSG3 $(12 \times 12)$ & 11.1020 & & 6.2837 & 0.99 & 6.2135 & 0.9940 \\
\hline & CS-DSG3 $(24 \times 24)$ & 11.3055 & 1.1645 & 6.3672 & 1.0113 & 6.2969 & 1.0053 \\
\hline \multirow{5}{*}{10} & Reddy and Liu FSL & 12.1230 & 3.5760 & 6.6247 & 2.4109 & 6.5595 & 2.4030 \\
\hline & DSG & .0923 & 3.5495 & 7 & 2.4 & 6.5701 & 009 \\
\hline & CS-DSG3 $(8 \times 8)$ & 11.5641 & 3.4124 & 6.3912 & 2.0314 & 6.3294 & 2.2933 \\
\hline & CS-DSG3 $(12 \times 12)$ & 11.9266 & & & 2.3 & 861 & 649 \\
\hline & CS-DSG3 $(24 \times 24)$ & 12.1165 & 3.5625 & 6.6612 & 2.4123 & 6.5886 & 2.4041 \\
\hline \multirow{5}{*}{100} & & 12.3700 & & & 4.3026 & & 4.3021 \\
\hline & DSG3 $(24 \times 24)$ & 12.3911 & 10.465 & 6.7438 & 4.3438 & 6.6640 & 4.3391 \\
\hline & CS-DSG3 $(8 \times 8)$ & 11.8434 & 9.7343 & 6.4817 & 4.0756 & 6.4092 & 4.0730 \\
\hline & CS-DSG3 $(12 \times 12)$ & 12.2177 & 10.277 & 6.6518 & 4.2516 & 6.5786 & 4.2507 \\
\hline & CS-DSG3 $(24 \times 24)$ & 12.4478 & 10.522 & 6.7564 & 4.3453 & 6.6828 & 4.3447 \\
\hline
\end{tabular}


uniformly distributed and point load obtained by the CS-DSG3 are listed in Tabs. 2 and 3 along with those by Reddy and Liu [10] using the FSDT, respectively. The various ratios of values side-to-thickness and values of radius-to-thickness are also examined. It is seen that the results by the CS-DSG3 are softer than those of the DSG3 and agree well with those published by Reddy and Liu [10] using the FSDT.

Table 2. The non-dimensional center deflections of the laminated spherical shells under uniform load

\begin{tabular}{|c|c|c|c|c|c|c|c|}
\hline \multirow{2}{*}{$R / a$} & \multirow{2}{*}{ Theory } & \multicolumn{2}{|c|}{$0^{\circ} / 90^{\circ}$} & \multicolumn{2}{|c|}{$0^{\circ} / 90^{\circ} / 0^{\circ}$} & \multicolumn{2}{|c|}{$0^{\circ} / 90^{\circ} / 90^{\circ} / 0^{\circ}$} \\
\hline & & \multicolumn{2}{|c|}{$a / h=10 a / h=100$} & \multicolumn{4}{|c|}{$a / h=10 a / h=100 a / h=10 a / h=100$} \\
\hline \multirow{4}{*}{5} & Reddy and Liu FSDT [10] & 19.9440 & 1.7535 & 9.7937 & 1.5118 & 9.8249 & 1.5358 \\
\hline & CS-DSG3 $(8 \times 8)$ & 16.8807 & 1.6712 & 9.3469 & 1.4427 & 9.3365 & 1.4630 \\
\hline & CS-DSG3 $(12 \times 12)$ & 17.3799 & 1.6926 & 9.5609 & 1.4628 & 9.5678 & 1.4856 \\
\hline & CS-DSG3 $(24 \times 24)$ & 17.6854 & 1.7059 & 9.6914 & 1.4762 & 9.7095 & 1.4995 \\
\hline \multirow{4}{*}{10} & Reddy and Liu FSDT [10] & 19.0650 & 5.5428 & 10.110 & 3.6445 & 10.141 & 3.7208 \\
\hline & CS-DSG3 $(8 \times 8)$ & 18.2055 & 5.3245 & 9.7984 & 3.5237 & 9.7879 & 3.5780 \\
\hline & CS-DSG3 $(12 \times 12)$ & 18.7586 & 5.4562 & 10.025 & 3.6008 & 10.032 & 3.6713 \\
\hline & CS-DSG3 $(24 \times 24)$ & 19.0971 & 5.5213 & 10.164 & 3.6454 & 10.186 & 3.7221 \\
\hline \multirow{4}{*}{100} & Reddy and Liu FSDT [10] & 19.4640 & 16.6450 & 10.2180 & 6.6421 & 10.2490 & 6.6772 \\
\hline & CS-DSG3 $(8 \times 8)$ & 18.6726 & 15.5468 & 9.95190 & 6.3613 & 9.94110 & 6.4508 \\
\hline & CS-DSG3 $(12 \times 12)$ & 19.2457 & 16.3981 & 10.1839 & 6.5904 & 10.1908 & 6.7124 \\
\hline & CS-DSG3 $(24 \times 24)$ & 19.5964 & 16.7744 & 10.3253 & 6.7133 & 10.3436 & 6.8493 \\
\hline
\end{tabular}

Table 3. The non-dimensional center deflections of the laminated spherical shells under central concentrated load

\begin{tabular}{|c|c|c|c|c|c|c|c|}
\hline \multirow{2}{*}{$R / a$} & \multirow{2}{*}{ Theory } & \multicolumn{2}{|c|}{$0^{\circ} / 90^{\circ}$} & \multicolumn{2}{|c|}{$0^{\circ} / 90^{\circ} / 0^{\circ}$} & \multicolumn{2}{|c|}{$0^{\circ} / 90^{\circ} / 90^{\circ} / 0^{\circ}$} \\
\hline & & \multicolumn{6}{|c|}{$a \overline{a / h=10 a / h=100} a / h=10 a / h=100 a / h=10 a / h=100$} \\
\hline \multirow{4}{*}{5} & Reddy and Liu FSDT [10] & 71.015 & - & 51.410 & - & 49.360 & - \\
\hline & CS-DSG3 $(8 \times 8)$ & 57.019 & 6.7337 & 38.123 & 5.8155 & 36.595 & 5.3842 \\
\hline & CS-DSG3 $(12 \times 12)$ & 60.573 & 7.3182 & 41.223 & 6.2661 & 39.461 & 5.7427 \\
\hline & CS-DSG3 $(24 \times 24)$ & 65.315 & 7.8136 & 45.653 & 6.6565 & 43.682 & 6.0465 \\
\hline \multirow{4}{*}{10} & Reddy and Liu FSDT [10] & 73.836 & - & 52.273 & - & 50.186 & - \\
\hline & CS-DSG3 $(8 \times 8)$ & 60.590 & 16.625 & 39.479 & 11.927 & 37.906 & 11.122 \\
\hline & CS-DSG3 $(12 \times 12)$ & 64.279 & 17.456 & 42.636 & 12.645 & 40.823 & 11.652 \\
\hline & CS-DSG3 $(24 \times 24)$ & 69.145 & 18.078 & 47.133 & 13.176 & 45.109 & 12.049 \\
\hline \multirow{4}{*}{100} & Reddy and Liu FSDT [10] & 74.940 & - & 52.666 & - & 50.565 & - \\
\hline & CS-DSG3 $(8 \times 8)$ & 61.833 & 43.141 & 39.930 & 19.481 & 38.345 & 18.515 \\
\hline & CS-DSG3 $(12 \times 12)$ & 65.578 & 45.176 & 43.109 & 20.727 & 41.281 & 19.438 \\
\hline & CS-DSG3 $(24 \times 24)$ & 70.489 & 46.333 & 47.624 & 21.504 & 45.589 & 20.020 \\
\hline
\end{tabular}




\subsubsection{Laminated cylindrical shell}

Next, the static analysis of the laminated cylindrical shells is considered. All of the layers are made by the same material with mechanical properties given by: $E_{1}=$ $19.2 \times 10^{6} \mathrm{Psi} ; E_{2}=1.56 \times 10^{6} \mathrm{Psi}, G_{12}=G_{13}=0.82 \times 10^{6} \mathrm{Psi}, G_{23}=0.523 \times 10^{6} \mathrm{Psi}_{12} v_{12}=$ 0.24. The shell is subjected to a sinusoidal distributed load. Tab. 4 presents the nondimensional center deflections of the shell in comparison with those published by Khdeir et al. [9]. Despite using a coarse mesh $(12 \times 12)$, it is observed that the obtained results match well with exact solution by Khdeir et al. [9].

Table 4. The non-dimensional center deflections of the laminated cylindrical shells under sinusoidal distributed load

\begin{tabular}{clccc}
\hline$R / a(a / h=10)$ & \multicolumn{1}{c}{ Theory } & $0^{\circ} / 90^{\circ}$ & $0^{\circ} / 90^{\circ} / 0^{\circ}$ & $0^{\circ} / 90^{\circ} / 90^{\circ} / 0^{\circ}$ \\
\hline & FSDT [9] & 1.5614 & 0.8999 & - \\
5 & CS-DSG3 $(8 \times 8)$ & 1.4780 & 0.9090 & 0.8993 \\
& CS-DSG3 $(12 \times 12)$ & 1.5261 & 0.9359 & 0.9261 \\
& CS-DSG3 $(24 \times 24)$ & 1.5555 & 0.9525 & 0.9426 \\
\hline \multirow{5}{*}{10} & FSDT [9] & 1.5910 & 0.9434 & - \\
& CS-DSG3 $(8 \times 8)$ & 1.5257 & 0.9269 & 0.9175 \\
& CS-DSG3 $(12 \times 12)$ & 1.5756 & 0.9545 & 0.9450 \\
& CS-DSG3 $(24 \times 24)$ & 1.6062 & 0.9714 & 0.9618 \\
\hline \multirow{3}{*}{50} & FSDT [9] & 1.6000 & 0.9583 & - \\
& CS-DSG3 $(8 \times 8)$ & 1.5420 & 0.9327 & 0.9234 \\
& CS-DSG3 $(12 \times 12)$ & 1.5926 & 0.9605 & 0.9511 \\
& CS-DSG3 $(24 \times 24)$ & 1.6235 & 0.9776 & 0.9681 \\
\hline
\end{tabular}

\subsection{Free vibration analysis}

In this example, the free vibration analysis of simply supported laminated composite spherical and cylindrical shells is considered. All layers of these shells are assumed to be of the same thickness and material with mechanical properties given by: $E_{1}=25 E_{2}$, $G_{12}=0.5 E_{2}, G_{13}=0.5 E_{2}, G_{23}=0.2 E_{2}, v_{12}=0.25$. The non-dimensional frequencies of the laminated spherical shell by the CS-DSG3 are compared with those by Reddy and Liu [10] in Tab. 5. Tab. 6 contains non-dimensional frequencies of the laminated cylindrical shell which are compared with analytical solutions by Reddy and Liu [10]. The shape of the first-six mode shapes of these shells by the CS-DSG3 is also displayed in Figs. 3 and 4 . It can be seen that the results obtained by the CS-DSG3 agree well with reference solutions using FSDT of Reddy and Liu [10]. 
Table 5. Non-dimensional frequencies $\bar{\omega}$ of a cross-ply laminated spherical shells $\left(R_{x}=R_{y}=R\right)$

\begin{tabular}{|c|c|c|c|c|c|c|c|}
\hline \multirow{2}{*}{$R / a$} & \multirow{2}{*}{ Theory } & \multicolumn{2}{|c|}{$0^{\circ} / 90^{\circ}$} & \multicolumn{2}{|c|}{$0^{\circ} / 90^{\circ} / 0^{\circ}$} & \multicolumn{2}{|c|}{$0^{\circ} / 90^{\circ} / 90^{\circ} / 0^{\circ}$} \\
\hline & & $a / h=10$ & $a / h=100$ & $a / h=10$ & $a / h=100$ & $a / h=10$ & $a / h=100$ \\
\hline \multirow{4}{*}{5} & FSDT [10] & 9.2309 & 28.8250 & 12.3720 & 30.9930 & 12.4370 & 12.3800 \\
\hline & CS-DSG3 $(8 \times 8)$ & 9.6105 & 30.0054 & 12.7958 & 32.2573 & 12.8746 & 32.3533 \\
\hline & CS-DSG3 $(12 \times 12)$ & 9.3569 & 29.3434 & 12.4863 & 31.5120 & 12.5614 & 31.6050 \\
\hline & CS-DSG3 $(24 \times 24)$ & 9.2078 & 28.9614 & 12.3037 & 31.0822 & 12.3766 & 31.1728 \\
\hline \multirow{4}{*}{10} & FSDT [10] & 8.9841 & 16.7060 & 12.2150 & 20.3470 & 12.4370 & 20.3800 \\
\hline & CS-DSG3 $(8 \times 8)$ & 9.3713 & 17.4354 & 12.6673 & 21.2329 & 12.7437 & 21.2675 \\
\hline & CS-DSG3 $(12 \times 12)$ & 9.1206 & 16.9796 & 12.3583 & 20.6578 & 12.4314 & 20.6921 \\
\hline & CS-DSG3 $(24 \times 24)$ & 8.9734 & 16.7380 & 12.1762 & 20.3421 & 12.2474 & 20.3764 \\
\hline \multirow{4}{*}{100} & FSDT [10] & 8.9009 & 9.7896 & 12.1630 & 15.2440 & 12.2280 & 15.2450 \\
\hline & CS-DSG3 $(8 \times 8)$ & 9.2911 & 10.358 & 12.6241 & 16.0094 & 12.6998 & 16.0110 \\
\hline & CS-DSG3 $(12 \times 12)$ & 9.0412 & 9.9574 & 12.3152 & 15.4827 & 12.3878 & 15.4837 \\
\hline & CS-DSG3 $(24 \times 24)$ & 8.8946 & 9.7703 & 12.1333 & 15.2047 & 12.2040 & 15.2057 \\
\hline
\end{tabular}

Table 6. The non-dimensional $\bar{\omega}$ frequencies of a cross-ply laminated cylindrical shells $\left(R_{y}=R, R_{x}=\infty\right)$

\begin{tabular}{|c|c|c|c|c|c|c|c|}
\hline \multirow{2}{*}{$R / a$} & \multirow{2}{*}{ Theory } & \multicolumn{2}{|c|}{$0^{\circ} / 90^{\circ}$} & \multicolumn{2}{|c|}{$0^{\circ} / 90^{\circ} / 0^{\circ}$} & \multicolumn{2}{|c|}{$0^{\circ} / 90^{\circ} / 90^{\circ} / 0^{\circ}$} \\
\hline & & $a / h=10$ & $a / h=100$ & $a / h=10$ & $a / h=100$ & $a / h=10$ & $a / h=100$ \\
\hline \multirow{4}{*}{5} & FSDT [10] & 8.9082 & 16.6680 & 12.2070 & 20.332 & 12.2670 & 20.3610 \\
\hline & Present $(8 \times 8)$ & 9.2805 & 17.1638 & 12.4794 & 20.9195 & 12.5887 & 20.9538 \\
\hline & Present $(12 \times 12)$ & 9.0985 & 16.8699 & 12.2934 & 20.5549 & 12.3868 & 20.5866 \\
\hline & Present $(24 \times 24)$ & 9.0123 & 16.7363 & 12.2064 & 20.3824 & 12.2883 & 20.4132 \\
\hline \multirow{4}{*}{10} & FSDT [10] & 8.8879 & 11.831 & 12.1730 & 16.6250 & 12.2360 & 16.6340 \\
\hline & Present $(8 \times 8)$ & 9.3048 & 12.4510 & 12.6379 & 17.4240 & 12.7141 & 17.4355 \\
\hline & Present $(12 \times 12)$ & 9.0550 & 12.0455 & 12.3289 & 16.8880 & 12.4019 & 16.8991 \\
\hline & Present $(24 \times 24)$ & 8.9084 & 11.8461 & 12.1468 & 16.6014 & 12.2180 & 16.6126 \\
\hline \multirow{4}{*}{100} & FSDT [10] & 8.8974 & 9.7108 & 12.1630 & 15.1980 & 12.2270 & 15.1990 \\
\hline & Present $(8 \times 8)$ & 9.2853 & 10.280 & 12.6222 & 15.9617 & 12.6969 & 15.9618 \\
\hline & Present $(12 \times 12)$ & 9.0356 & 9.8801 & 12.3134 & 15.4353 & 12.3849 & 15.4348 \\
\hline & Present $(24 \times 24)$ & 8.8891 & 9.6935 & 12.1315 & 15.1576 & 12.2011 & 15.1571 \\
\hline
\end{tabular}




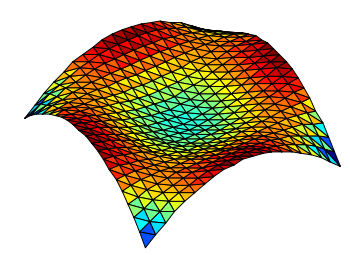

Mode 1

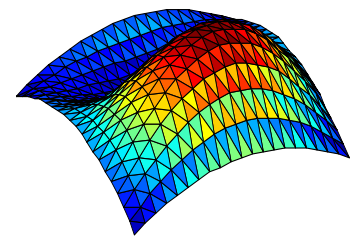

Mode 4

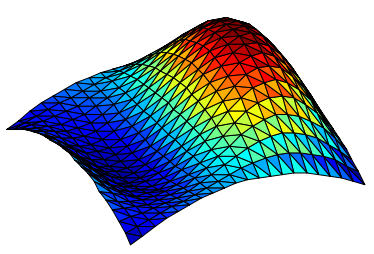

Mode 2

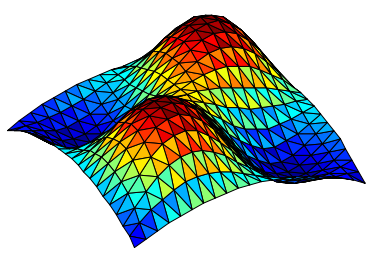

Mode 5

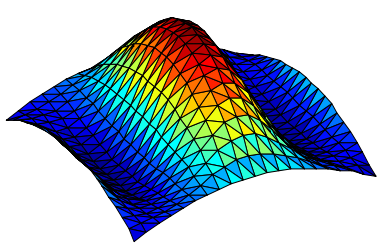

Mode 3

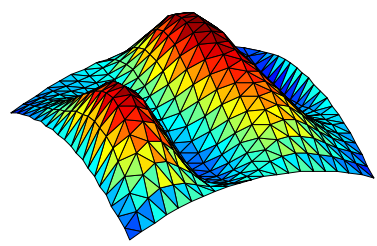

Mode 6

Fig. 3. The first six mode shapes of the laminated spherical shell by CS-DSG3

$\left(R / a=10, a / h=10,0^{\circ} / 90^{\circ} / 0^{\circ}\right)$

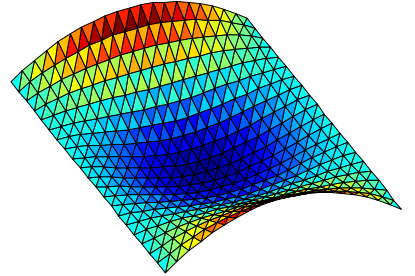

Mode 1

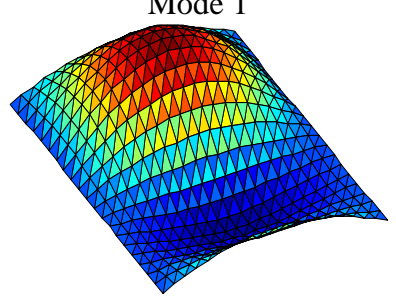

Mode 4

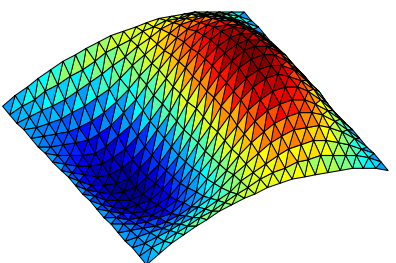

Mode 2

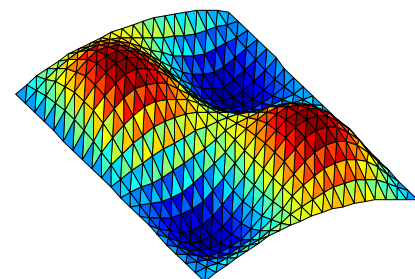

Mode 5

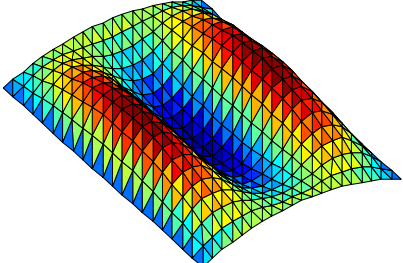

Mode 3

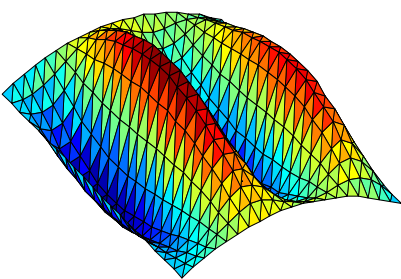

Mode 6

Fig. 4. The first six mode shapes of the laminated cylindrical shell by CS-DSG3

$$
\left(R / a=10, a / h=100,0^{\circ} / 90^{\circ} / 0^{\circ}\right)
$$

\section{CONCLUSIONS}

The paper presents an extension of the CS-DSG3 using the FSDT for static and free vibration analyses of laminated composite shells. Through the present formulations and obtained numerical results, some main points can be withdrawn as:

i). The CS-DSG3 uses three-node triangular elements that are easier generated automatically for arbitrary complex geometrical domains.

ii). The CS-DSG3 uses only minimum degrees of freedom at each vertex node, so we can expect an efficient analysis in term of computational cost. The CS-DSG3 is free of shear locking for laminated composite shells. 
iii). Due to using the gradient smoothing technique which can help soften the overstiff behavior in the DSG3, the proposed CS-DSG3 improves significantly the accuracy of the numerical results and has a good convergence performance.

iv). The accuracy and reliability of the CS-DSG3 are verified by comparing its numerical solutions with those of other available numerical results. The results by the CS-DSG3 agree well with all reference solutions in different analyses.

The method presented herein is promising to be an effectively alternative method of classical finite elements for analysis of laminated composite shells in practice.

\section{ACKNOWLEDGEMENTS}

This research is funded by Vietnam National Foundation for Science and Technology Development (NAFOSTED) under grant number 107.02-2017.08.

\section{REFERENCES}

[1] A. Bhimaraddi. Free vibration analysis of doubly curved shallow shells on rectangular planform using three-dimensional elasticity theory. International Journal of Solids and Structures, 27, (7), (1991), pp. 897-913. doi:10.1016/0020-7683(91)90023-9.

[2] A. Bhimaraddi. Three-dimensional elasticity solution for static response of orthotropic doubly curved shallow shells on rectangular planform. Composite Structures, 24, (1), (1993), pp. 67-77. doi:10.1016/0263-8223(93)90056-v.

[3] K. P. Rao. A rectangular laminated anisotropic shallow thin shell finite element. Computer Methods in Applied Mechanics and Engineering, 15, (1), (1978), pp. 13-33. doi:10.1016/00457825(78)90003-8.

[4] J. N. Reddy. Exact solutions of moderately thick laminated shells. Journal of Engineering Mechanics, 110, (5), (1984), pp. 794-809. doi:10.1061/(asce)0733-9399(1984)110:5(794).

[5] S. J. Hossain, P. K. Sinha, and A. H. Sheikh. A finite element formulation for the analysis of laminated composite shells. Computers E Structures, 82, (20-21), (2004), pp. 1623-1638. doi:10.1016/j.compstruc.2004.05.004.

[6] D. Chakravorty, J. N. Bandyopadhyay, and P. K. Sinha. Free vibration analysis of pointsupported laminated composite doubly curved shells - A finite element approach. Computers E Structures, 54, (2), (1995), pp. 191-198. doi:10.1016/0045-7949(94)00329-2.

[7] J. N. Reddy. Mechanics of laminated composite plates and shells: Theory and analysis. CRC Press, (2004).

[8] L. Librescu, A. A. Khdeir, and D. Frederick. A shear deformable theory of laminated composite shallow shell-type panels and their response analysis I: Free vibration and buckling. Acta Mechanica, 76, (1-2), (1989), pp. 1-33. doi:10.1007/bf01175794.

[9] A. A. Khdeir, L. Librescu, and D. Frederick. A shear deformable theory of laminated composite shallow shell-type panels and their response analysis II: Static response. Acta Mechanica, 77, (1-2), (1989), pp. 1-12. doi:10.1007/bf01379740.

[10] J. N. Reddy and C. F. Liu. A higher-order shear deformation theory of laminated elastic shells. International Journal of Engineering Science, 23, (3), (1985), pp. 319-330. doi:10.1016/0020-7225(85)90051-5.

[11] R. K. Khare, T. Kant, and A. K. Garg. Closed-form thermo-mechanical solutions of higherorder theories of cross-ply laminated shallow shells. Composite Structures, 59, (3), (2003), pp. 313-340. doi:10.1016/s0263-8223(02)00245-3. 
[12] M. Y. Yasin and S. Kapuria. An efficient layerwise finite element for shallow composite and sandwich shells. Composite Structures, 98, (2013), pp. 202-214. doi:10.1016/j.compstruct.2012.10.048.

[13] G. Giunta, F. Biscani, S. Belouettar, and E. Carrera. Hierarchical modelling of doubly curved laminated composite shells under distributed and localised loadings. Composites Part B: Engineering, 42, (4), (2011), pp. 682-691. doi:10.1016/j.compositesb.2011.02.002.

[14] A. J. M. Ferreira, E. Carrera, M. Cinefra, and C. M. C. Roque. Analysis of laminated doublycurved shells by a layerwise theory and radial basis functions collocation, accounting for through-the-thickness deformations. Computational Mechanics, 48, (1), (2011), pp. 13-25. doi:10.1007/s00466-011-0579-4.

[15] C. H. Thai, H. Nguyen-Xuan, N. Nguyen-Thanh, T.-H. Le, T. Nguyen-Thoi, and T. Rabczuk. Static, free vibration, and buckling analysis of laminated composite Reissner-Mindlin plates using NURBS-based isogeometric approach. International Journal for Numerical Methods in Engineering, 91, (6), (2012), pp. 571-603. doi:10.1002/nme.4282.

[16] C. H. Thai, A. J. M. Ferreira, S. P. A. Bordas, T. Rabczuk, and H. Nguyen-Xuan. Isogeometric analysis of laminated composite and sandwich plates using a new inverse trigonometric shear deformation theory. European Journal of Mechanics-A/Solids, 43, (2014), pp. 89-108. doi:10.1016/j.euromechsol.2013.09.001.

[17] C. H. Thai, H. Nguyen-Xuan, S. P. A. Bordas, N. Nguyen-Thanh, and T. Rabczuk. Isogeometric analysis of laminated composite plates using the higher-order shear deformation theory. Mechanics of Advanced Materials and Structures, 22, (6), (2015), pp. 451-469. doi:10.1080/15376494.2013.779050.

[18] T. Rabczuk, P. M. A. Areias, and T. Belytschko. A meshfree thin shell method for non-linear dynamic fracture. International Journal for Numerical Methods in Engineering, 72, (5), (2007), pp. 524-548. doi:10.1002/nme.2013.

[19] T. Rabczuk, R. Gracie, J.-H. Song, and T. Belytschko. Immersed particle method for fluidstructure interaction. International Journal for Numerical Methods in Engineering, 81, (1), (2010), pp. 48-71. doi:10.1002/nme.2670.

[20] N. Nguyen-Thanh, J. Kiendl, H. Nguyen-Xuan, R. Wüchner, K.-U. Bletzinger, Y. Bazilevs, and T. Rabczuk. Rotation free isogeometric thin shell analysis using PHT-splines. Computer Methods in Applied Mechanics and Engineering, 200, (47-48), (2011), pp. 3410-3424. doi:10.1016/j.cma.2011.08.014.

[21] N. Nguyen-Thanh, N. Valizadeh, M. N. Nguyen, H. Nguyen-Xuan, X. Zhuang, P. Areias, G. Zi, Y. Bazilevs, L. De Lorenzis, and T. Rabczuk. An extended isogeometric thin shell analysis based on Kirchhoff-Love theory. Computer Methods in Applied Mechanics and Engineering, 284, (2015), pp. 265-291. doi:10.1016/j.cma.2014.08.025.

[22] G. R. Liu and T. Nguyen-Thoi. Smoothed finite element methods. CRC Press, Taylor and Francis Group, NewYork, (2010).

[23] G. R. Liu, K. Y. Dai, and T. T. Nguyen. A smoothed finite element method for mechanics problems. Computational Mechanics, 39, (6), (2007), pp. 859-877. doi:10.1007/s00466-006-00754.

[24] G. R. Liu, T. Nguyen-Thoi, H. Nguyen-Xuan, K. Y. Dai, and K. Y. Lam. On the essence and the evaluation of the shape functions for the smoothed finite element method (SFEM). International Journal for Numerical Methods in Engineering, 77, (13), (2009), pp. 1863-1869. doi:10.1002/nme.2587.

[25] T. T. Nguyen, G. R. Liu, K. Y. Dai, and K. Y. Lam. Selective smoothed finite element method. Tsinghua Science \& Technology, 12, (5), (2007), pp. 497-508. doi:10.1016/s1007-0214(07)70125-6. 
[26] G. R. Liu, H. Nguyen-Xuan, and T. Nguyen-Thoi. A theoretical study on the smoothed FEM (S-FEM) models: Properties, accuracy and convergence rates. International Journal for Numerical Methods in Engineering, 84, (10), (2010), pp. 1222-1256. doi:10.1002/nme.2941.

[27] G. R. Liu, T. T. Nguyen, K. Y. Dai, and K. Y. Lam. Theoretical aspects of the smoothed finite element method (SFEM). International Journal for Numerical Methods in Engineering, 71, (8), (2007), pp. 902-930. doi:10.1002/nme.1968.

[28] D. T. Hau, N. T. M. Hanh, and N. T. Trung. A cell-based smoothed discrete shear gap method (CS-FEM-DSG3) for dynamic response of laminated composite plate subjected to blast loading. Vietnam Journal of Mechanics, 37, (2), (2015), pp. 81-90. doi:10.15625/08667136/37/2/5019.

[29] G. R. Liu, T. Nguyen-Thoi, H. Nguyen-Xuan, and K. Y. Lam. A node-based smoothed finite element method (NS-FEM) for upper bound solutions to solid mechanics problems. Computers \& Structures, 87, (1-2), (2009), pp. 14-26. doi:10.1016/j.compstruc.2008.09.003.

[30] T. Nguyen-Thoi, G. R. Liu, H. Nguyen-Xuan, and C. Nguyen-Tran. Adaptive analysis using the node-based smoothed finite element method (NS-FEM). International Journal for Numerical Methods in Biomedical Engineering, 27, (2), (2011), pp. 198-218. doi:10.1002/cnm.1291.

[31] T. Nguyen-Thoi, G. R. Liu, and H. Nguyen-Xuan. Additional properties of the node-based smoothed finite element method (NS-FEM) for solid mechanics problems. International Journal of Computational Methods, 6, (04), (2009), pp. 633-666. doi:10.1142/s0219876209001954.

[32] G. R. Liu, T. Nguyen-Thoi, and K. Y. Lam. An edge-based smoothed finite element method (ES-FEM) for static, free and forced vibration analyses of solids. Journal of Sound and Vibration, 320, (4-5), (2009), pp. 1100-1130. doi:10.1016/j.jsv.2008.08.027.

[33] T. Nguyen-Thoi, G. R. Liu, and H. Nguyen-Xuan. An n-sided polygonal edge-based smoothed finite element method (nES-FEM) for solid mechanics. International Journal for Numerical Methods in Biomedical Engineering, 27, (9), (2011), pp. 1446-1472. doi:10.1002/cnm.1375.

[34] T. Nguyen-Thoi, G. R. Liu, K. Y. Lam, and G. Y. Zhang. A face-based smoothed finite element method (FS-FEM) for 3D linear and geometrically non-linear solid mechanics problems using 4-node tetrahedral elements. International journal for numerical methods in Engineering, 78, (3), (2009), pp. 324-353. doi:10.1002/nme.2491.

[35] T. Nguyen-Thoi, P. Phung-Van, H. Luong-Van, H. Nguyen-Van, and H. Nguyen-Xuan. A cellbased smoothed three-node Mindlin plate element (CS-MIN3) for static and free vibration analyses of plates. Computational Mechanics, 51, (1), (2013), pp. 65-81. doi:10.1007/s00466012-0705-y.

[36] X. Y. Cui, G. R. Liu, G. Y. Li, X. Zhao, T. Nguyen-Thoi, and G. Y. Sun. A smoothed finite element method (SFEM) for linear and geometrically nonlinear analysis of plates and shells. Comput Model Eng Sci, 28, (2), (2008), pp. 109-125.

[37] C. H. Thai, L. V. Tran, D. T. Tran, T. Nguyen-Thoi, and H. Nguyen-Xuan. Analysis of laminated composite plates using higher-order shear deformation plate theory and nodebased smoothed discrete shear gap method. Applied Mathematical Modelling, 36, (11), (2012), pp. 5657-5677. doi:10.1016/j.apm.2012.01.003.

[38] H. H. Phan-Dao, H. Nguyen-Xuan, C. Thai-Hoang, T. Nguyen-Thoi, and T. Rabczuk. An edge-based smoothed finite element method for analysis of laminated composite plates. International Journal of Computational Methods, 10, (01), (2013). doi:10.1142/s0219876213400057.

[39] H. Nguyen-Xuan, G. R. Liu, C. Thai-Hoang, and T. Nguyen-Thoi. An edge-based smoothed finite element method (ES-FEM) with stabilized discrete shear gap technique for analysis of 
Reissner-Mindlin plates. Computer Methods in Applied Mechanics and Engineering, 199, (9-12), (2010), pp. 471-489. doi:10.1016/j.cma.2009.09.001.

[40] T. Nguyen-Thoi, T. Bui-Xuan, P. Phung-Van, S. Nguyen-Hoang, and H. Nguyen-Xuan. An edge-based smoothed three-node Mindlin plate element (ES-MIN3) for static and free vibration analyses of plates. KSCE Journal of Civil Engineering, 18, (4), (2014), pp. 1072-1082. doi:10.1007/s12205-014-0002-8.

[41] H. Nguyen-Xuan, T. Rabczuk, N. Nguyen-Thanh, T. Nguyen-Thoi, and S. Bordas. A nodebased smoothed finite element method with stabilized discrete shear gap technique for analysis of Reissner-Mindlin plates. Computational Mechanics, 46, (5), (2010), pp. 679-701. doi:10.1007/s00466-010-0509-x.

[42] T. Nguyen-Thoi, T. Bui-Xuan, P. Phung-Van, H. Nguyen-Xuan, and P. Ngo-Thanh. Static, free vibration and buckling analyses of stiffened plates by CS-FEM-DSG3 using triangular elements. Computers \& Structures, 125, (2013), pp. 100-113. doi:10.1016/j.compstruc.2013.04.027.

[43] T. Nguyen-Thoi, P. Phung-Van, C. Thai-Hoang, and H. Nguyen-Xuan. A cell-based smoothed discrete shear gap method (CS-DSG3) using triangular elements for static and free vibration analyses of shell structures. International Journal of Mechanical Sciences, 74, (2013), pp. 32-45. doi:10.1016/j.ijmecsci.2013.04.005.

[44] K.-U. Bletzinger, M. Bischoff, and E. Ramm. A unified approach for shear-locking-free triangular and rectangular shell finite elements. Computers $\mathcal{E}$ Structures, 75, (3), (2000), pp. 321334. doi:10.1016/s0045-7949(99)00140-6.

[45] O. C. Zienkiewicz and R. L. Taylor. The finite element method for solid and structural mechanics. Butterworth-Heinemann, (2005).

[46] T. Nguyen-Thoi, P. Phung-Van, H. Nguyen-Xuan, and C. Thai-Hoang. A cell-based smoothed discrete shear gap method using triangular elements for static and free vibration analyses of Reissner-Mindlin plates. International Journal for Numerical Methods in Engineering, 91, (7), (2012), pp. 705-741. doi:10.1002/nme.4289. 